\title{
BEAM STABILITY STUDIES AND IMPROVEMENTS AT ALADDIN*
}

\author{
W. S. Trzeciak, M. A. Green, and the SRC Staff, \\ Synchrotron Radiation Center, 3731 Schneider Dr., Stoughton, WI, 53589-3097, USA
}

Abstract

Recent improvements in beam position stability at Aladdin, the $1 \mathrm{GeV}$ electron storage ring at the Synchrotron Radiation Center, are reported. Stabilizing the beam position monitors (BPM's), in conjunction with the use of a global feedback system, keeps the beam position within $\pm 3 \mu \mathrm{m}$ of its starting value during a user fill. Relocation of transformers and the dipole power supply choke have made substantial reductions in the 60 and $720 \mathrm{~Hz}$ motions of the electron beam, allowing a broader scope to the infrared experimental program. Work on stabilizing the synchrotron radiation source characteristics under various operating conditions is presented. Studies of weak resonances near the standard operating tune are leading to the use of closed loop tune control to maintain beam size stability. Lattice corrections employed during the scanning of undulators keep the source sizes fixed down to the $2 \%$ level. Future plans for improved optical beam position and size monitors, renovation of the BPM electronics, implementation of a hydrostatic leveling system, and temperature monitoring of the ring components is also presented.

\section{INTRODUCTION}

Aladdin is a $1 \mathrm{GeV}$ electron storage ring dedicated to synchrotron radiation. The University of Wisconsin, Madison, operates it under contract to the National Science Foundation. It has been in operation since 1985, and, in many respects, it performs at the level of third generation synchrotron radiation sources.

The ring-operating schedule has been unchanged for several years now [1,2]. Regular operation is 20 hours per day, 5 days per week, with the additional possibility of 4 hours of user beam every weekday morning or full day operation on weekends.

While Aladdin is a $1 \mathrm{GeV}$ storage ring, most of the operating time for user beams is at $800 \mathrm{MeV}$, starting out at about $250 \mathrm{~mA}$ and has a current-lifetime product of about $950 \mathrm{~mA} \cdot \mathrm{h}$. Typical measured source sizes from the bending magnets are $\sigma_{x}=450 \mu \mathrm{m}$, and $\sigma_{y}=85 \mu \mathrm{m}$, whereas calculated source sizes in the middle of long straight sections (undulator source points) are $\sigma_{\mathrm{x}}=814 \mu \mathrm{m}$, and $\sigma_{\mathrm{y}}=72 \mu \mathrm{m}$.

\footnotetext{
* Work supported by the NSF grant DMR-95-31009.

walter@src.wisc.edu
}

\section{POSITION STABILITY}

\subsection{General}

Several improvements in the structure of the vacuum chamber have been made over the last few years. New stripline pairs, used as beam position monitors (BPM's), have been installed in the whole ring, except for one final long straight section. The new monitors are thicker and more robust; there is essentially no warpage during bakeout or when heated by synchrotron radiation. The monitors are mounted in chamber sections that pass through quadrupoles that are now uncoupled, via a bellows, from the downstream dipole vacuum chamber section. In the past, small changes in the dipole section position, e.g. to reset a photon-beamline port, would change the BPM position in the upstream quadrupole doublet. This no longer happens. Since the BPM's are now mechanically uncoupled from the dipole sections, they are now firmly clamped to the quadrupole bases, which are mounted, via short steel pedestals, to the bedrock under the floor.

Calibration of the BPM's is accomplished by the usual current-shunting technique. Most BPM's are located at quarupoles. While 1 or $2 \%$ of the current is alternately shunted and not shunted around a quadrupole, the beam is steered until the closed orbit is invariant. At that point the BPM reading is the calibration offset.

Some of the ring quadrupoles have back-leg windings that are used as dipole steering corrections. Since we've mechanically uncoupled the chamber from its adjacent quadrupole, we've measured a large difference in calibration (as much as $164 \mu \mathrm{m}$ ) comparing with and without current in the "piggy backed" dipole steering. Non-linear effects of the uncorrected orbit traversing the BPM at a large angle may explain this.

Prior to the upgrade one of each stripline pair also doubled as an ion-clearing electrode. The new chamber sections have a separate ion clearing strip, simplifying the electrical hookup of the BPM's and eliminating a potential source of noise in the BPM electronics.

\subsection{Long Term}

Over the length of a user fill, 4 to 8 hours, the orbit is stable to within $\pm 3 \mu \mathrm{m}$. In order to get this level of stability, the Global Feedback system is active for all user beams. This system simply reoptimizes the orbit to a 
target orbit at a $2 \mathrm{~Hz}$ rate. Day to day orbit position repeatability is about $\pm 6 \mu \mathrm{m}$. Three photon monitors record the beam position: two photodiode arrays and a CCD camera.

Recognizing that the long-term beam position stability is affected by the positional stability of the ring components, (including the BPM's, both beam driven and optical) two measurement systems are being installed on the ring. We are placing 20 (soon to add 80 more) computer readable temperature sensors on various ring components: e.g. quadrupole stands and dipole stands. Changes in temperature that produce ring component position changes, especially in the vertical direction, will be monitored and correlated with beam position variation.

A hydrostatic leveling system (HLS) has been acquired. This is the same type of system used at ESRF. Once the system is tested and installed, the relative vertical position of ring components will be constantly monitored. The resolution of the system is at the $1 \mu \mathrm{m}$ level, with an accuracy $\leq 5 \mu \mathrm{m}$. It appears that some resolution is lost because of the need to move the sensing electronics away from the sensing head. This was necessary because the combined sensor and electronics is not meant to work in even modest external magnetic fields.

Another hydrostatic problem that we are just starting to investigate is the presence of ground water in the bedrock under the concrete floor that supports the ring. Shortly after a large water spill, several photon beamlines required realignment, indicating that the stored beam position had somehow been changed as a result of the water seepage under the floor.

As indicated above, Aladdin regularly runs at either $800 \mathrm{MeV}$ or $1 \mathrm{GeV}$. The central orbit is in a different position at the two energies, causing some user beamlines to require either readjustment or, for some high resolution beamlines, simply to avoid using the $1 \mathrm{GeV}$ beam altogether. Accompanying the change in position there is an excessively large increase in vertical beam size and a change in beam rotation of $6^{\circ}$. The size and rotation variations are consistent with changes in the ring coupling. Measurements have shown that the coupling varies almost linearly from 0.013 at $800 \mathrm{MeV}$ to 0.029 at $1 \mathrm{GeV}$. Original dipole field data show the magnets "shorten" at fields corresponding to $1 \mathrm{GeV}$ operation. This combined decrease in $\int \mathrm{B}_{\mathrm{y}} \mathrm{ds}$ and increase in coupling may be the cause of the different orbit, but there are still many unanswered questions. For example, why there are differences between BPM calibrations at the two energies, as much as $160 \mu \mathrm{m}$.

\subsection{Short Term}

In the realm of fast changes in beam position, frequencies faster than a few hertz, many problems have been identified and solved. The infrared beamlines are very sensitive to beam motion at the ac power line frequency, $60 \mathrm{~Hz}$, and its harmonics. The main ring dipole power supply has a 12-phase rectifier circuit, and horizontal beam motions at $720 \mathrm{~Hz}$ were quite large (See Figure 1). The cause was a large series inductor (choke) that was located close, about $40 \mathrm{~cm}$, to the beam orbit. We relocated the choke and observed a large reduction in the $720 \mathrm{~Hz}$ horizontal beam motion (Figure 1).

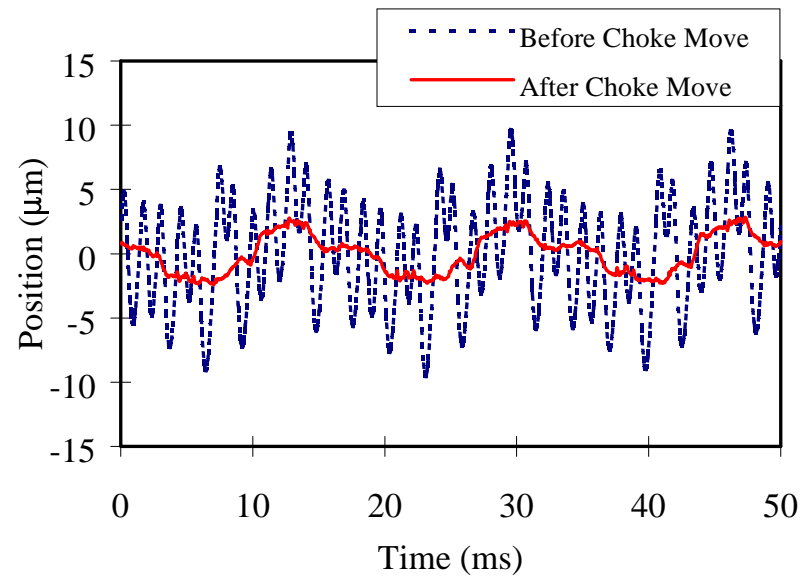

Figure 1: Reduction of $720 \mathrm{~Hz}$ Beam Motion

Figure 1 also shows residual $60 \mathrm{~Hz}$ beam motion. Several sources have been identified and either fixed or shortly will be fixed. The $60 \mathrm{~Hz}$ beam motion was reduced by a factor of 10 after four ac service transformers were relocated from adjacent to the beam orbit to about $3 \mathrm{~m}$ away [2]. Replacing the ac solenoids with a dc type removed the $60 \mathrm{~Hz}$ noise contribution of the ring sector valves. However, the actuators of the beamline front-end valves are also known to be a source of $60 \mathrm{~Hz}$ oscillations and will be replaced later this year. Also, the main rf system will be tested to verify that there is not some $60 \mathrm{~Hz}$ modulation that is affecting beam motion.

To reduce the $60 \mathrm{~Hz}$ beam motion even more, several improvements are scheduled. The main ring dipole magnet power supply will get a transistor passbank (there is none now) and each of the quadrupole and dipole power supplies will have new controllers, all with digital signal processors (DSP's).

\section{SIZE STABILITY}

\subsection{General}

Section 1 notes that a typical vertical beam size at a dipole source point is $85 \mu \mathrm{m}$. This size can vary depending on the level of coupling in the ring. Two operational regimes should be distinguished. The first is how the ring is set for users at the start of a user experimental run. At this time four skew quadrupoles around the ring can provide some control of beam size and rotation, and they are used to set beam size to a standard value. This can only set the beam size within 
rather narrow limits, however, and more skew quadrupoles would be required to provide better-localized size control.

The main cause of variability in beam size during a user fill is the scanning of uncorrected undulators. The correction scheme used for each undulator is to shunt some current around the upstream and downstream vertically focusing quadrupoles. The amount of current shunted is a function of the peak undulator field. The main residual correction is almost entirely correction of the vertical tune. With this scheme the tune can be kept constant, but the smallest average vertical beam size variation observed during scanning is $2 \%$. For many users, especially those employing experiments that rely on the difference between the above and below median plane polarization of the radiation, vertical beam size changes of $1 \%$ are too large. We are continuing to look at ways to further reduce these beam size changes. The most promising avenues are choosing a different operating point, keeping the global tune constant, dynamic control of skew quadrupoles, or, most likely, a combination of the above.

\subsection{Long Term}

Over the course of a user fill the beam size doesn't vary by more than $\pm 3 \mu \mathrm{m}$, with most of the variation occurring in the first $2 \mathrm{~h}$, most likely from the well known effect of high current on beam size. From one fill to the next the starting beam size is dependent on the ring tunes and the initial state of the three undulators in regular use. The machine operators normalize the beam size at one optical monitor by varying skew quadrupole settings. This can and does lead to too much beam size variation at other sources around the ring. The normal operating point is relatively close to several weak resonances.

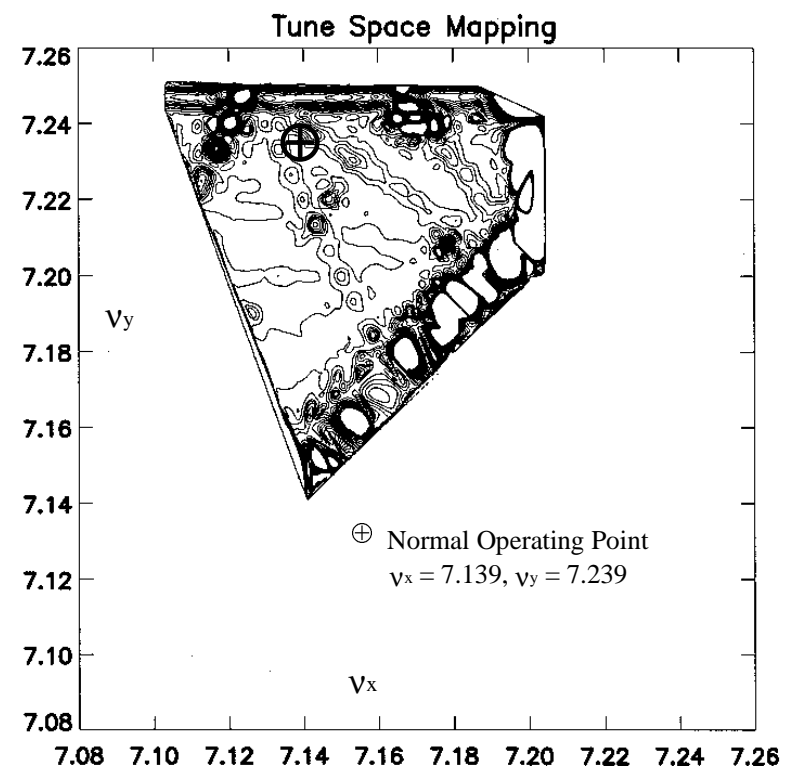

Figure 2: Tune Space near Operating Point
Figure 2 shows a beam size mapping of the tune space around Aladdin's operating point. At that point small changes in ring parameters, even scanning of wellcompensated undulators, can cause small beam size changes. Under investigation is a permanent move to a different operating point. In the near future, usage of a software feedback loop to keep the tunes constant will become a normal mode of operation.

\section{IMPROVEMENT PLANS}

\subsection{Diagnostic Stations}

Besides the tune control, temperature monitors, and HLS mentioned above, four new diagnostic stations are in the design stage. These stations, one in each corner of the ring, will be dedicated to measurements of beam size, rotation, and position in both planes. Each station will have a CCD camera to look at the beam cross section and measure beam size. Also, each station will have a position sensitive device (PSD) to measure the position of the beam down to the $1 \mu \mathrm{m}$ level. At least one of the stations will have additional linear photodiode arrays to look for high frequency beam motion. A prototype of the optical design has been tested, and the $45^{\circ}$ mirrors that take the light into the stations have been procured. The stations should be operational by the end of the year.

\subsection{BPM Electronics}

Modern advancements in electronics have seen the increased use of digital signal processors (DSP's). To make use of this modern technology the electronics that measures beam position will be completely rebuilt to utilize DSP's. This will increase the resolution of the BPM system by a factor of 10 .

\section{REFERENCES}

[1] D. L. Huber, et al, 'SRC: Current activities and plans for the future', Nuclear Instruments and Methods in Physics Research A 347 (1994) 49-51.

[2] W. S. Trzeciak and Staff, 'Aladdin, Present and Future', Proceedings of the 1997 Particle Accelerator Conference, Vancouver, B. C., Pg. 865. 